\title{
Efficient micromachining of poly(vinylidene fluoride) using a laser-plasma EUV source
}

\author{
A. Bartnik · H. Fiedorowicz • R. Jarocki • J. Kostecki • \\ M. Szczurek • P.W. Wachulak
}

Received: 5 October 2011 / Accepted: 20 October 2011 / Published online: 11 November 2011

(c) The Author(s) 2011. This article is published with open access at Springerlink.com

\begin{abstract}
In this paper an efficient micromachining of poly(vinylidene fluoride) (PVDF) by direct photo-etching with a laser-plasma EUV (extreme ultraviolet) source was demonstrated for the first time. Mass spectroscopy was employed to investigate the ablation products and revealed emission of numerous molecular species of C-containing fragments of the polymer chain. Chemical surface changes after irradiation were investigated using X-ray photoelectron spectroscopy (XPS). The XPS spectra obtained for PVDF samples, irradiated with low and high EUV fluence, indicate significant differences between chemical structures in nearsurface layers. It was shown that irradiation with low fluence results in defluorination and thus carbon enrichment of the polymer in near-surface layer. In contrary, irradiation with high fluence leads to intense material ablation and hardly modifies the chemical structure of the remaining material.
\end{abstract}

\section{Introduction}

Poly(vinylidene fluoride) (PVDF) is an important fluoropolymer because of its piezoelectric, pyroelectric and ferroelectric properties. It is also known to have an extremely high chemical stability and electrical resistivity. Micro- or even nanopatterning of PVDF is highly desirable for applications in multifunctional and integrated devices. Many works have been performed on surface processing of PVDF using ion beams $[1,2]$ synchrotron X-ray [3, 4] and UV laser

A. Bartnik $(\bowtie) \cdot$ H. Fiedorowicz · R. Jarocki · J. Kostecki .

M. Szczurek · P.W. Wachulak

Institute of Optoelectronics, Military University of Technology,

Kaliskiego 2, 00-908 Warsaw, Poland

e-mail: abartnik@wat.edu.pl

Fax: +48-22-6668950 radiation [5, 6]. Irradiation of PVDF with these sources resulted in strong modification of the molecular structure in a near-surface layer of the polymer. The chemical changes are associated with dehydrogenation, defluorination $[2,4,7,8]$ and cross-linking between the polymer chains $[4,9]$. The changes, especially the defluorination, strongly influence the ablation process. In case of utilizing the synchrotron radiation for photomicromachining the maximum achievable etch depth was $\sim 10 \mu \mathrm{m}$ due to a saturation behavior [4]. QMS (quadrupole mass spectrometry) showed that only $\mathrm{H}_{2}$, $\mathrm{F}$ and HF gaseous species were emitted from the surface during irradiation $[3,4]$. The removal of the fluorine atoms resulted in decrease of the irradiated region volume, hence, was responsible for the photomachining mechanism. Simultaneously the F fraction in PVDF decreased gradually as the etching progressed and finally the process stopped. Similar behavior was reported for PVDF irradiated with ion beams $[1,2]$ and excimer lasers [5-7].

In this work micromachining of PVDF by using a laserplasma EUV (extreme ultraviolet) source was demonstrated for the first time. It was shown that $\mathrm{C}$-containing molecules were emitted from the polymer during irradiation, which is contrary to the experiments mentioned above. The ablation rate in this case was comparable to EUV ablation rate of polytetrafluoroethylene (PTFE) and fluorinated ethylenepropylene (FEP) [11].

\section{Experiment}

In our experiments, a 10-Hz laser-plasma EUV source, based on a double-stream gas-puff target, irradiated with the 3-ns/0.8 J Nd:YAG laser pulse, was used. The target was created by pulsed injection of a krypton-xenon (90/10\%) mixture gas into a hollow stream of helium by employing 
electromagnetic valve system equipped with a double nozzle set-up. The focusing conditions and plasma parameters were adjusted to obtain maximum intensity in the EUV spectral region. The radiation was focused using a gold-plated grazing incidence ellipsoidal collector, manufactured in Reflex s.r.o., (at present: Rigaku Innovative Technologies Europe s.r.o.), Czech Republic. The collector allowed for effective focusing of radiation emitted from $\mathrm{Kr} / \mathrm{Xe}$ plasma in the wavelength range $\lambda=9-70 \mathrm{~nm}$. The most intense emission was in the relatively narrow spectral region centered at $\lambda=11 \pm 1 \mathrm{~nm}$. The spectral intensity at longer wavelength range was much smaller, however, the spectrally integrated intensities in both ranges were comparable. The EUV fluence in the focal plane of the collector exceeded $60 \mathrm{~mJ} / \mathrm{cm}^{2}$ in the center of the focal spot. FWHM diameter of the intensity distribution in the focal spot was $1.4 \mathrm{~mm}$. Detailed description of the source and parameters of the focused EUV radiation can be found elsewhere [10].

PVDF foils of 50- $\mu$ m thickness from Goodfellow, utilized in this experiment, were used without any treatment. The samples were mounted on the XYZ motorized translation stage, placed in the EUV collector focal plane and irradiated through a contact metallic mask with square orifices $60 \times 60 \mu \mathrm{m}^{2}$ in size. Irradiation was performed at $10-\mathrm{Hz}$ repetition rate with different exposure times up to $2 \mathrm{~min}$.

\section{Results}

After irradiation the polymer samples were investigated using a scanning electron microscope (SEM). For better visualization of the microstructures, produced in PVDF, the samples were tilted horizontally and scanned at $60^{\circ}$ angle in respect to the sample surface. SEM images of the microstructures obtained with different magnifications showing the central area of the focal spot after 1 min exposure are presented in Fig. 1. It can be noticed that both the walls and the bottoms of the etched structure are smooth and the etched depth is comparable to the PVDF foil thickness. Irradiation with 700 EUV pulses was sufficient to etch through the foil in the central part of the focal spot. It means that the average ablation rate for the EUV fluence $60 \mathrm{~mJ} / \mathrm{cm}^{2}$ was approximately $70 \mathrm{~nm} /$ pulse. No visible darkening of the surface was observed in the irradiated area suggesting lack of carbonization. The surface darkening was visible, however, for the fluence below $10 \mathrm{~mJ} / \mathrm{cm}^{2}$.

The ablation process was investigated using a quadrupole mass spectrometry (QMS). The measurements were performed with a QMG 700 mass spectrometer having a detection limit of about $10^{-15} \mathrm{mbar}$ and mass range 1-512 amu. The mass spectra, obtained during irradiation of the PVDF samples, consisted of multiple peaks corresponding to both the ablation products and background components. The
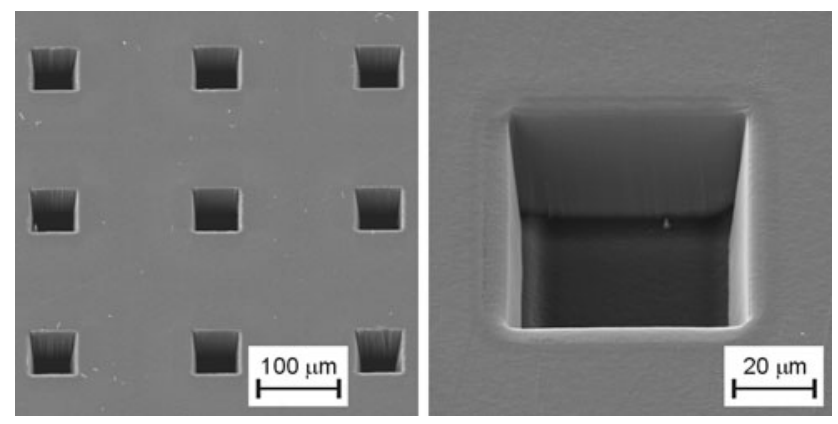

Fig. 1 Microstructures obtained in a $50 \mu \mathrm{m}$ thick PVDF foil as a result of 1 min EUV irradiation through a contact mask

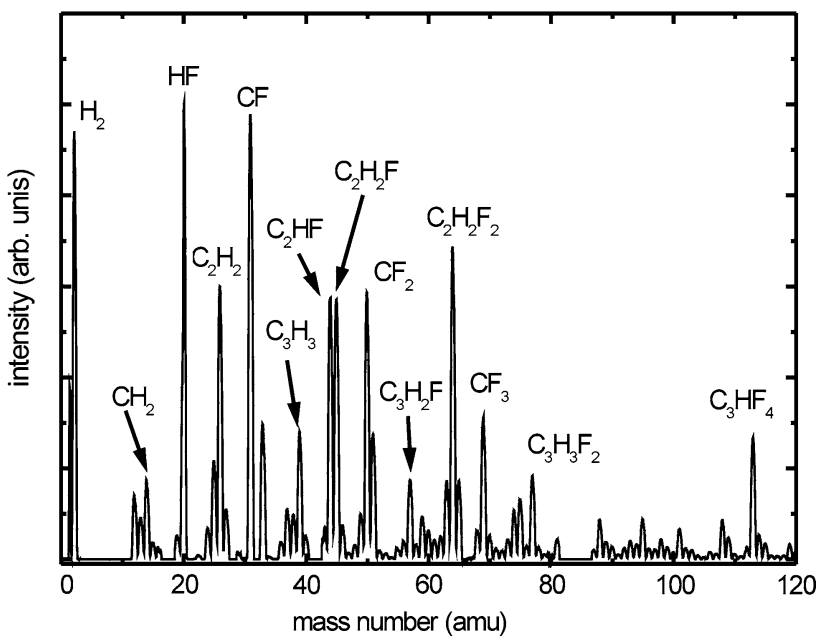

Fig. 2 QMS spectrum of PVDF irradiated with EUV pulses in the focal plane of the EUV collector

background mass spectrum obtained for the working EUV source, without irradiation of the polymer sample, revealed the presence of $\mathrm{H}_{2}, \mathrm{H}_{2} \mathrm{O}, \mathrm{CO} / \mathrm{N}_{2}$, and $\mathrm{CO}_{2}$ molecules, which are expected in a typical clean vacuum system and additionally $\mathrm{Kr}$, Xe and He atoms, used for the gas-puff target formation. The background spectrum was subtracted from the spectra obtained during irradiation of the PVDF samples. A typical spectrum of the ablation products is presented in Fig. 2. The spectrum consists of multiple peaks corresponding to decomposition products of the irradiated polymer. As could be expected from the irradiation experiments using synchrotrons there are two intense peaks corresponding to the $\mathrm{H}_{2}$ and $\mathrm{HF}$ molecules [3, 4]. Our results, however, are significantly different with respect to these measurements because of the presence of many other peaks corresponding to the molecules or radicals containing $\mathrm{C}$ atoms. Such peaks are not present in the spectra obtained with synchrotron irradiation. Apart from that intensities of $\mathrm{H}_{2}$ and HF peaks are similar in our case while $\mathrm{H}_{2}$ emission in case of X-ray irradiation is over 3 times larger comparing to emission of HF. In case of EUV irradiation intensity of a peak corresponding to $\mathrm{CF}$ radical is the same as the $\mathrm{H}_{2}$ peak, intensities of 

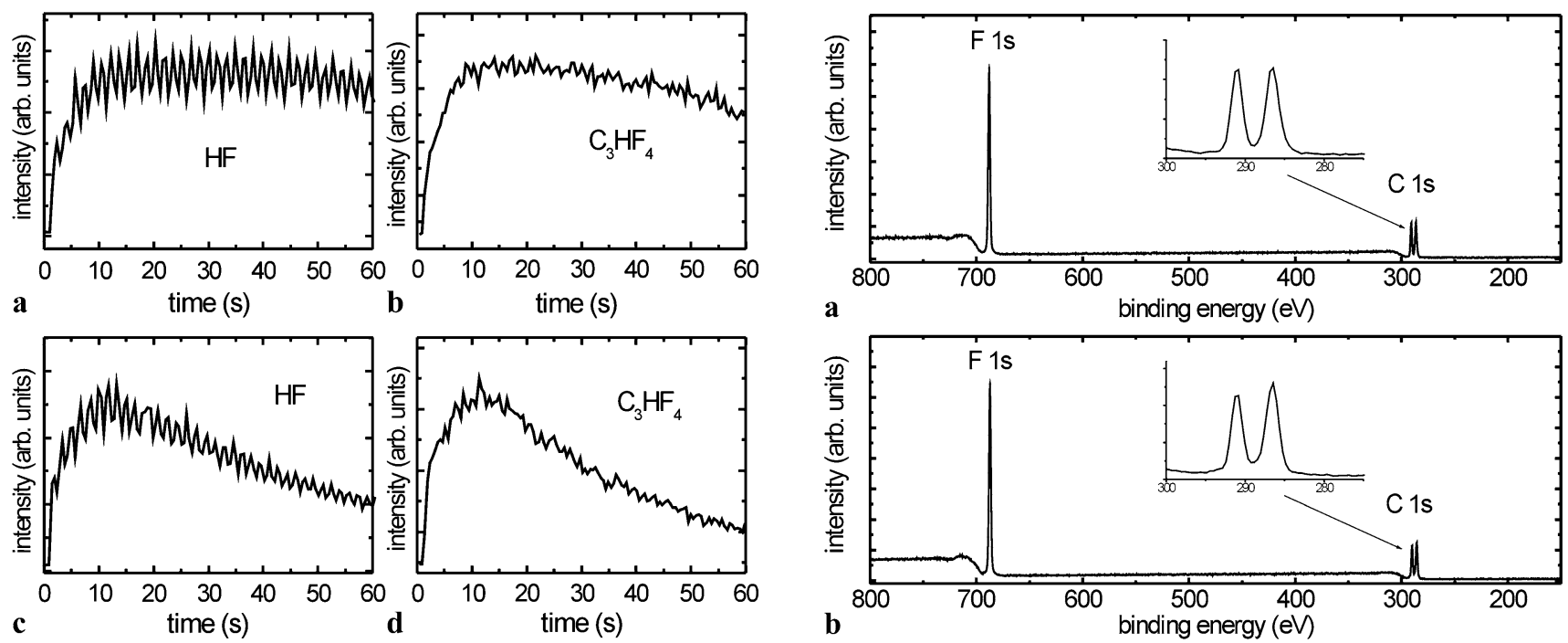

Fig. 3 Time evolutions of $\mathrm{HF}$ and $\mathrm{C}_{3} \mathrm{HF}_{4}$ emission during 1 min irradiation with EUV pulses: $(\mathbf{a}, \mathbf{b})$ in the focal plane of the EUV collector, (c, d) $10 \mathrm{~mm}$ downstream the focal plane

other peaks, corresponding to the presence of C-containing molecules, are lower but still very well defined. Their spectrally integrated intensities are about 4 times higher in relation to the $\mathrm{H}_{2}$ and $\mathrm{HF}$ peaks. The mass spectrum revealed the presence of numerous molecular species containing one to three $\mathrm{C}$ atoms in the mass range up to $120 \mathrm{amu}$. These are radicals or unsaturated molecules being fragments of the polymer chain. The $\mathrm{C}: \mathrm{H}: \mathrm{F}$ ratio obtained from the spectrum is 1:1:0.9, respectively, which is in good agreement with the ratio in PVDF molecules (1:1:1). It means that an average atomic composition of the ablated material is similar to the atomic composition of the polymer and thus there should be no significant changes in chemical composition of the material remained on the polymer surface after the ablation.

In Fig. 3 the time evolution of $\mathrm{HF}$ and $\mathrm{C}_{3} \mathrm{HF}_{4}$ emission during EUV irradiation of PVDF is presented. The $\mathrm{C}_{3} \mathrm{HF}_{4}$ was chosen as a representative of C-containing ablation products having a typical time evolution. The time evolution was investigated in the focal plane of the EUV collector (Fig. 3a, b) and $10 \mathrm{~mm}$ downstream the focal plane (Fig. 3c, d) where the EUV fluence was less than $10 \mathrm{~mJ} / \mathrm{cm}^{2}$ and the darkening of the surface after irradiation occurred. It can be noticed that after 1 min EUV exposure of the PVDF sample located in the focal plane, there was only a relatively small decrease of $\mathrm{HF}(10 \%)$ and $\mathrm{C}_{3} \mathrm{HF}_{4}(20 \%)$ emission, while in case of irradiation with low fluence, the decrease was much higher $60 \%$ and $65 \%$, respectively. Initial increase of the QMS signals corresponding to $\mathrm{HF}$ and $\mathrm{C}_{3} \mathrm{HF}_{4}$ emission is typical for QMS measurements in the experimental arrangement employed in this work. Time duration of the increase is associated with approaching an equilibrium between emission rate of the decomposition products and their removal by the pumping system. In case of constant emis-

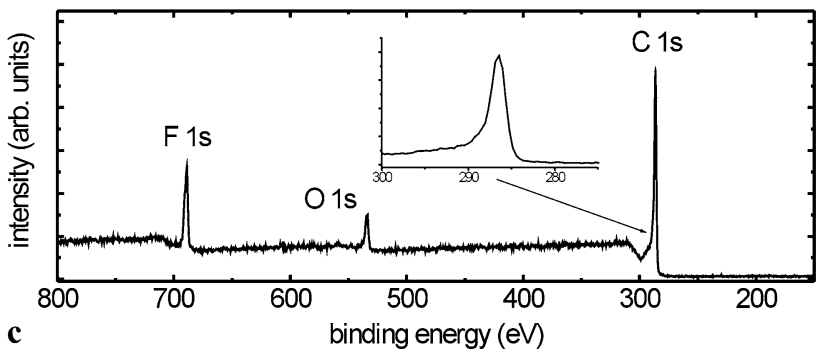

Fig. 4 XPS spectra of PVDF: (a) pristine sample, (b) remaining surface material after ablation (450 EUV pulses, high fluence), (c) chemically modified material in the near-surface layer (450 EUV pulses, low fluence)

sion rate the signal would reach a plateau at some level and remain constant until the irradiation stops. Decrease of the QMS signal during irradiation is associated with decrease of emission rate of the decomposition products. In case of constant irradiation parameters such decrease of the emission rate can be connected only with changes of the photochemical properties of the irradiated polymer. The changes are confirmed by darkening of the polymer surface irradiated with low fluence. Such darkening reported in [3] was assigned to formation of a carbon-rich layer acting as a barrier for further etching and preventing the diffusion of etched gaseous products. Fast decrease of emission of the decomposition products in case of low fluence suggests carbon enrichment in the irradiated layer. Carbon enrichment was also reported in our previous works using an X-ray photoemission spectroscopy (XPS) [12, 13]. In case of irradiation in the focal plane, where the fluence is the highest, surface darkening is limited to the periphery of the focal spot. It suggests that carbon enrichment occurs only in this area and results in smaller decrease of emission of the decomposition products.

Chemical changes in the near-surface layer of PVDF were additionally investigated using X-ray photoelectron spectroscopy (XPS). The XPS spectra were acquired for 
polymer samples exposed to different number of EUV pulses with low and high fluence. For comparison the XPS measurements were performed also for a pristine PVDF sample. Typical XPS spectra obtained for the pristine sample and samples irradiated with 450 EUV pulses are presented in Fig. 4. In all cases two characteristic features corresponding to photoelectrons coming from fluorine (F1s) and carbon $(\mathrm{C} 1 \mathrm{~s})$ atoms are present. Their relative intensities in case of the untreated polymer sample and the sample irradiated with high fluence are almost identical. The relative concentrations of $\mathrm{F} / \mathrm{C}$ atoms calculated from the peak intensities, taking into account the corresponding weights, are $50 / 50 \%$ and $49 / 51 \%$, respectively. These concentrations are similar to theoretical values. It means that the polymer material that remains on the surface after ablation is chemically unaffected. Completely different is the situation in case of PVDF irradiated with low fluence. In this case the $\mathrm{C} 1$ s peak is much stronger comparing to $\mathrm{F} 1 \mathrm{~s}$ peak. The calculated fluorine concentration decreased to $12.5 \%$ while carbon concentration increased to $79.7 \%$. Additionally form of the $\mathrm{C} 1 \mathrm{~s}$ peak acquired with high resolution differs significantly from $\mathrm{C} 1 \mathrm{~s}$ peaks obtained for the pristine sample. The high resolution $\mathrm{C} 1 \mathrm{~s}$ spectrum of untreated PVDF contains two dominating peaks corresponding to $-\mathrm{CH}_{2}$ - (binding energy $286.44 \mathrm{eV}$ ) and $-\mathrm{CF}_{2}-$ (binding energy $290.9 \mathrm{eV}$ ). In case of the polymer irradiated with low fluence the peak corresponding to binding energy $290.9 \mathrm{eV}$ strongly decreased. It means that irradiation of PVDF with low fluence results in carbon enrichment in the near-surface layer, similarly as in case of X-ray irradiation [3, 4]. An additional O1s peak corresponds to $7.8 \%$ of oxygen incorporated at the polymer surface exposed to air after irradiation.

Similar results of XPS measurements were obtained using a $\mathrm{KrF}$ excimer laser for irradiation of PVDF [5]. In this case the fluorine concentration on the polymer surface decreased after irradiation to about $40 \%$ according to the initial value. In our experiment decrease of the fluorine concentration reached 36, 25, $19 \%$ for 150,450 and 600 EUV pulses, respectively. Irradiation with low fluence EUV pulses gives then similar effect as $\mathrm{KrF}$ laser. There is, however, significant difference concerning irradiation fluence: surface carbonization using $\mathrm{KrF}$ laser requires at least $140 \mathrm{~mJ} / \mathrm{cm}^{2}$ while in case of EUV the fluence should not be higher than $10 \mathrm{~mJ} / \mathrm{cm}^{2}$. For PVDF samples irradiated with X-rays UPS measurements of the polymer valence band were performed. Also in this case strong decrease of peak corresponding to fluorine content in the near-surface layer was reported [3,4].

Different behavior of PVDF irradiated with EUV pulses against the response of the polymer to X-ray and UV radiation is likely associated with the unique properties of the EUV radiation in respect to the other radiation sources. The EUV photons are strongly absorbed in any polymer (absorption depth of 10-100 nm in PVDF). Energy of a single
EUV photon is sufficient to excite or release any electron from the valence band. Each of these photoelectrons has enough energy to excite several electronic states. The relative probabilities of triplet states formation are greatly enhanced with respect to excitation with electromagnetic radiation. It means that radiationless de-excitation processes, including bond breaking, are predominant without preferences regarding the type of bond $(\mathrm{C}-\mathrm{C}, \mathrm{C}-\mathrm{F}, \mathrm{C}-\mathrm{H})$. Similar mechanism is responsible for interaction of X-rays with PVDF but the absorption depth in this case is much larger $(10 \mu \mathrm{m}-$ $1 \mathrm{~mm}$ ) and the exposure time is much longer-up to several hours, which results in the power density several order of magnitude lower compared to the EUV irradiation. Apart from that, C-containing fragments of the polymer chain, produced deep inside the polymer, can be too large to diffuse to the surface. Thus mainly $\mathrm{H}_{2}$, and HF molecules are emitted changing the chemical structure and the composition of the polymer chains. In case of irradiation of PVDF with the UV lasers the power density in a single pulse is comparable to that with the EUV irradiation. The mechanism of interaction, however, is different. Energy of the UV photon is sufficient to break a single $\mathrm{C}-\mathrm{F}$ bond and, in consequence, allows formation of the HF molecule. The HF molecule is being released from the polymer surface and a $\mathrm{C}-\mathrm{C}$ bond is converted to the $\mathrm{C}=\mathrm{C}$ bond [7]. Further cross-linking leads to the formation of benzene rings and finally graphite crystals on the polymer surface.

\section{Summary}

The laser-plasma EUV source was used for micromachining of PVDF by direct photo-etching for the first time. Efficient material ablation was obtained in case of EUV irradiation with the high fluence $\left(60 \mathrm{~mJ} / \mathrm{cm}^{2}\right)$. Investigation of the ablation products with QMS demonstrated emission of C-containing fragments of the polymer chain. It was shown that the $\mathrm{C}: \mathrm{H}: \mathrm{F}$ ratio obtained from the spectrum, is in a good agreement with the stoichiometric composition of PVDF molecules. XPS spectra acquired for the polymer after ablation are almost identical to the spectrum of pristine PVDF, indicating preservation of the chemical structure of the remaining material. This way ablation process remains undisturbed during irradiation with consecutive EUV pulses. XPS measurements applied to the polymer irradiated with low fluence $\left(<10 \mathrm{~mJ} / \mathrm{cm}^{2}\right)$ indicate strong chemical modification in the near-surface layer. In this case defluorination and thus carbon enrichment in the surface material was revealed.

Acknowledgements This work was supported by the grant No. N N202 174939 of the Ministry of Science and Higher Education of Poland and was partially performed under COST Action MP0601. 
Open Access This article is distributed under the terms of the Creative Commons Attribution Noncommercial License which permits any noncommercial use, distribution, and reproduction in any medium, provided the original author(s) and source are credited.

\section{References}

1. L. Torrisi, G. Ciavola, R. Percolla, F. Benyaich, Nucl. Instrum. Methods Phys. Res. B 116, 473-477 (1996)

2. L. Torrisi, R. Percolla, Nucl. Instrum. Methods Phys. Res., Sect. B, Beam Interact. Mater. Atoms 117, 387-391 (1996)

3. H.M. Manohara, E. Morikawa, J. Choi, P.T. Sprunger, IEEE/ASME J. Microelectromech. Syst. 8, 417-422 (1999)

4. E. Morikawa, J. Choi, H.M. Manohara, J. Appl. Phys. 87, 40104016 (2000)

5. Y. Ji, Y. Jiang, Appl. Phys. Lett. 89, 221103 (2006)

6. Y. Liu, Y. Jiang, Opt. Express 18, 22041 (2010)
7. S.R. George, J.A. Leraas, S.C. Langford, J.T. Dickinson, Appl. Surf. Sci. 255, 9558-9561 (2009)

8. S.S. Chebotaryov, E.M. Baitinger, A.A. Volegov, I.G. Margamov, I.V. Gribov, N.A. Moskvina, V.L. Kuznetsov, S.E. Evsyukov, L.A. Pesin, Radiat. Phys. Chem. 75, 2024-2028 (2006)

9. M.D. Duca, C.L. Plosceanu, T. Pop, J. Appl. Polym. Sci. 67, 21252129 (1998)

10. A. Bartnik, H. Fiedorowicz, R. Jarocki, J. Kostecki, M. Szczurek, P.W. Wachulak, Nucl. Instrum. Methods Phys. Res. A 647, 125131 (2011)

11. A. Bartnik, H. Fiedorowicz, R. Jarocki, J. Kostecki, R. Rakowski, A. Szczurek, M. Szczurek, Acta Phys. Pol. A 117, 384-390 (2010)

12. A. Bartnik, H. Fiedorowicz, R. Jarocki, J. Kostecki, M. Szczurek, A. Biliński, O. Chernyayeva, J.W. Sobczak, Appl. Phys. A, Mater. Sci. Process. 99, 831-836 (2010)

13. A. Bartnik, H. Fiedorowicz, R. Jarocki, J. Kostecki, M. Szczurek, J. Electron Spectrosc. Relat. Phenom. (2011). doi:10.1016/j.elspec.2010.12.030 